\title{
Electrokinetic geophysics - a review
}

\author{
David Beamish and R. J. Peart \\ British Geological Survey, Keyworth, Nottingham NG12 5GG, U.K.
}

Beamish, D. \& Peart, R.J., 1998. Terra Nova, 10, 48-55.

DOI: 10.1046/j.1365-3121.1998.00160.x

\begin{abstract}
Electrokinetic phenomena occur in porous rocks and include streaming potential, electro-osmotic and electrophoretic effects. Geophysical techniques can generate acoustic body waves that stimulate electrokinetic coupling at subsurface boundaries. The electromagnetic effects generated are observed using surface electric field sensors. Improved methodologies, aided by recent modelling of the coupled acoustic and electromagnetic wave equations, are re-exploring electrokinetic behaviour in the near surface. The methodologies are aimed at providing geophysical tools that may characterize environmentally sensitive parameters such as the fluid content, the fluid geochemistry and the microstructural fabric of the subsurface.
\end{abstract}

\section{Introduction}

There is now a considerable and reawakening interest in the physics of electrokinetic phenomena within the subsurface. Such effects occur naturally, on a variety of time-scales, in all rock fluid systems (Corwin, 1990). Current interest is focused on artificially stimulating electrokinetic effects and making use of the fields and dynamic behaviour generated. The removal of contaminants from soils by electric fields is referred to as electrokinetic remediation (Probstein and Hicks, 1993). Typically a direct current is applied to sedimentary, near-surface rocks which induces osmotic movement of water away from a positively charged anode and toward a negatively charged cathode (Shapiro and Probstein, 1993). Ions and colloids migrate toward the electrode carrying an opposite charge and based on the results of laboratory tests and field trials (Lageman et al., 1989), electrokinetic remediation is a promising method for the recovery of ionic and water-soluble subsurface contaminants.

This review considers a different and recent use of electrokinetics, on far more rapid timescales, for the geophysical investigation of subsurface rock and fluid properties. In this non-invasive, surface sounding context a propagating pressure pulse is generated by a conventional acoustic (seismic) source. The propagating source generates streaming currents due to the pore-scale charges that exist in fluid-saturated porous rocks. Physical displacement of these charges at property interfaces causes a subsurface electromagnetic oscillation that can be detected by appropriate surface sensors. The properties involved in the coupling mechanism are a combination of the pore fluid properties (e.g. their electrical conductivity) and microstructural parameters (e.g. porosity and permeability). In addition to obtaining some knowledge of these properties, the simple detection of the presence or absence of groundwater would be a major benefit in many environments. 


\section{Historical background}

The idea that sound waves could generate electric fields was discussed in a paper in the first volume of the Journal of Chemical Physics by Debye (1933) in connection with a suspension of charged particles forming an electrolytic solution. Such 'electro-acoustic' techniques, applied to the determination of the electrokinetic potential (at ultrasound frequencies), have become traditional tools for the industrial/laboratory characterization of colloids and emulsions (O'Brien, 1988).

In the first volume of the journal Geophysics, Thompson (1936) pro- posed that the coupling of seismic and electric fields could be used as an exploration tool. The concept discussed was the variation in resistivity with elastic deformation and the term seismic electric effect was coined.

Ivanov (1939) made observations of electric fields generated by explosions. It was noted that there was a phase reversal in the electric field recorded when the elastic wave was generated on opposite sides of the electrode spread. The effect observed was dis-cussed in terms of the solid and liquid phases present in the rock mass. Electrokinetic phenomena were described in terms of charge separation at the 'double diffusion layer' of the pore space. The same studies and concepts as those presented by Ivanov (1939) are now being reinvestigated and are referred to here as ElectroKinetic (EK) geophysical sounding.

Potential applications of electro- acoustic measurements were noted, although the physics of the coupling mechanism between elastic and electromagnetic waves was poorly understood. Ivanov (1939) indeed surmised that electrical phenomena occurring in the Earth's crust during earthquakes are associated with such effects. This was later taken up by Mizutani et al. (1976) and Ishido and Mizutani (1981) who provide an experimental and theoretical basis of electrokinetic phenomena in rock-water systems and discuss possible earthquake-related effects.

Observational field studies of electrokinetic phenomena appear as 'intermittent' research papers in western journals in the 1950's (Martner and Sparks, 1959), the 1960's (Broding et al., 1963), the 1970's (Long and Rivers, 1975) and in the 1980's (Murty 1985). Soviet experiments are described by Parkhomenko (1971). The experiments include both laboratory and borehole investigations of electrokinetic effects (Parkhomenko and Gaskarov (1971). In 1993 two oil company researchers published the results of a systematic study of the potential of 'electroseismic effects' in the context of deep exploration for oil and gas (Thompson and Gist, 1993). The study, both theoretical and field based, predicted measurable electric field effects. The study concluded by suggesting the greatest potential for successful application lay in shallow exploration (e.g. for aquifers). In our studies which consider the potential of EK sounding for aquifer (and other) shallow investigations, the guidelines developed by Thompson and Gist (1993) have been followed. 


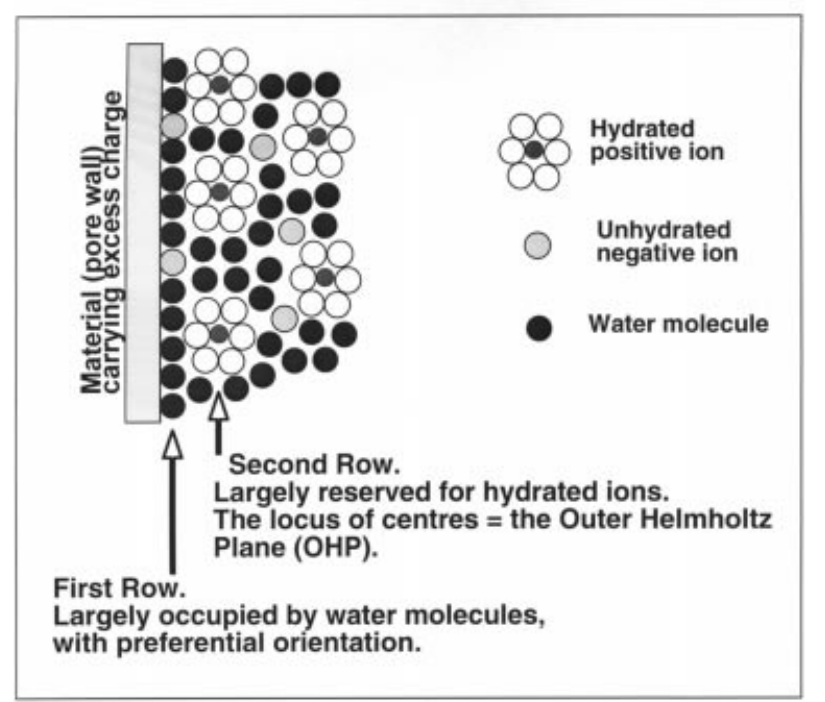

Figure 1. The Electrified Interface (after Bockris and Reddy, 1970). Schematic representation of the electrical double layer at a pore wall.

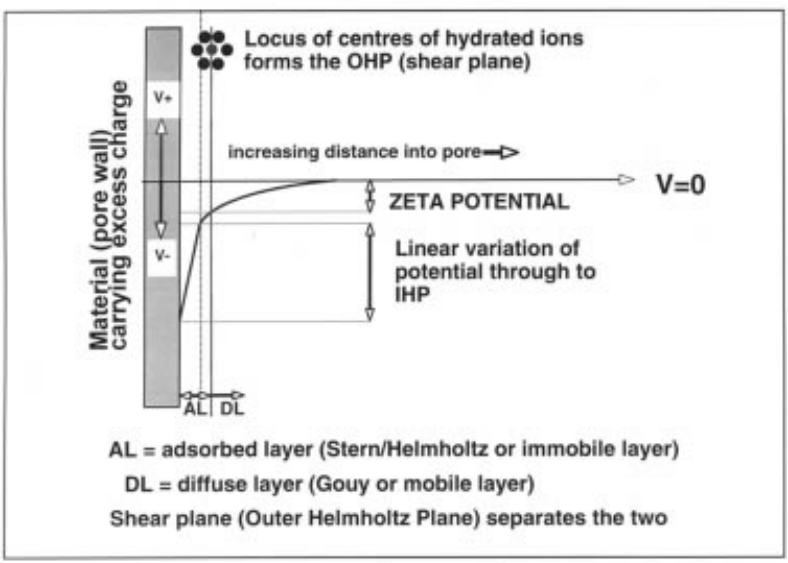

Figure 2. The Electrified Interface (after Bockris and Reddy, 1970). Schematic of the potential distribution across the electrical double layer.

\section{Porous rocks and streaming potentials}

Natural porous materials are formed by various minerals such as silicates, oxides and carbonates. Such minerals develop an electrical double layer (EDL) when in contact with an electrolyte (Bockris and Ready, 1970). The concept of the electrical double layer is illustrated in Fig. 1. The EDL is made up of a layer of ions adsorbed on the surface of the matrix and of a diffuse mobile layer extending into the liquid phase. An electrical (zeta) potential exists on the first non-bound plane along which interstitial pore fluid is capable of movement (Overbeek, 1952). The zeta potential is illustrated in Fig. 2. Typically the zeta potential may range from zero to values in excess of $150 \mathrm{mV}$ (Pride and Morgan, 1991) and constitutes stored energy in the porous rock-mass. Electrokinetic coupling occurs under non equilibrium conditions (e.g. when a pressure gradient is applied) and relative movement of the pore fluids results in a net displacement of charge across the EDL.

The relationship between differential pressure $(\Delta \mathrm{P})$ and resulting electrokinetic voltage $(\Delta V)$ is given by the Helmholtz-Schmoluchowski equation (Overbeek, 1952; Ishido and Mizutani, 1981): 
$\Delta \mathrm{V}=\mathrm{C} . \Delta \mathrm{P}$

where $\mathrm{C}$ is referred to as the streaming potential coefficient. The streaming potential coefficient has been studied in the context of other geophysical methods of exploration such as self-potential and streaming-potential (Corwin and Hoover, 1979); typical values (-12 to over $350 \mathrm{mV} / \mathrm{atm}$ ) in a variety of rock types are given in Ahmed (1964).

In the classical Helmholtz-Schmoluchowski equation, $\mathrm{C}$ is independent of any microstructural (pore) parameter and is given by:

$$
\mathrm{C}=\varepsilon \xi / \eta \sigma
$$

where, $\varepsilon, \xi, \eta$ and $\sigma$ are the fluid dielectric constant, the zeta potential, the fluid dynamic viscosity and the fluid electrical conductivity, respectively. The relationshipassumes (i) the pore hydraulic radius $(m)$ is $>>$ than the EDL thickness, (ii) flow is laminar (for large $m$ we expect nonlinear/turbulent flow and (iii) surface conductance is small ( $<<$ pore fluid volume conductance).

In situations in which electrical surface conduction becomes significant (e.g. decreasing pore size, low conductivity (fresh) fluids) the streaming potential is modified as:

$$
\mathrm{C}=\varepsilon \xi / \eta /\left(\sigma+\sigma_{\mathrm{sc}}\right)
$$

The additional term for surface conductivity $\left(\sigma_{\mathrm{sc}}\right)$ allows various micro-structural parameters of the pore space such as porosity and permeability to be introduced (e.g. Ishido and Mizutani, 1981). Jouniaux and Pozzi (1995) describe laboratory experiments on sandstone and limestone samples in which the streaming potential depends on sample permeability; the dependence is strong when fluid resistivity is high and vice versa. Laboratory experiments (Revil et al., 1996) indicate that the magnitude of the zeta potential, and hence streaming potential, increases with decreasing electrolyte concentration so that the largest streaming potentials are associated with fresh (nonsaline) fluid environments.

In the context of existing self-potential geophysics, seismoelectric experiments attempt to stimulate transitory electrokinetic coupling at seismic frequencies (typically $<500 \mathrm{~Hz}$ ). This contrasts sharply with the much slower, long-term natural processes (e.g. electrochemical and electrofiltration) involved in conventional self-potential applications.

\section{The acoustic source}

The theory for the propagation of the acoustic source follows the standard relationships of elastic wave propagation in a homogenous medium. An impulsive source, such as a hammer blow on a metal plate, propagating as a hemispherical wavefront with velocity $v=f \lambda$ (wherefisfrequency and $\lambda$ is wavelength) is assumed. Initially the source is wide-band and $f$ is multi-valued; frequencydependent absorption will eventually produce a wavelet of dominant $f$ and $\lambda$. One of the first elements of the wavefield to be examined is that connected with vertical propagation using ray theory.

The surface position of the source is referred to as the shot point. At a subsurface boundary, circular regions centered on the shot point are produced by the downgoing wave. As in the case of optics, the disturbances within even and odd numbered circular regions (Fresnel zones) with successive radii spaced one-half the wavelength apart are of opposite signs. The circular Fresnel zone, centered below 
the shot point is defined with approximate radius: $r=(z \lambda / 2)^{1 / 2}$ where $z$ is depth.

The concept of a downward-propagating sequence of first Fresnel zones, each capable of providing a radially coherent zone of displacement, is an important element of EK geophysical sounding. It is important first to note that in the case of a compressional acoustic wave propagating through a homogenous porous material, electrokinetic coupling produces a constant electric field confined to the wave (Fitterman, 1978; Pride and Haartsen, 1996). Independently propagating electromagnetic waves are not generated. When the acoustic wave traverses a boundary separating regions with different streaming potential coefficient then a charge separation that oscillates at the acoustic frequency is produced. This charge oscillation generates an electromagnetic field that can be observed at the surface. From the previous equations, the magnitude of the field will depend on the electrochemical properties of the rock/fluid and the mobility of the pore fluid. The subsurface property dependencies involved in electrokinetic coupling are not simple. The relationships suggest that all subsurface interfaces involving changes in the type of pore fluid (e.g. air or water), changes in rock type (giving rise to different zeta potentials), as well as microstructural properties (porosity and permeability) have to be considered.

In order to further understand the behaviour of electrokinetic phenomena, the fully coupled behaviour of elastic and electromagnetic wave interactions in porous media must be examined. The theories of quasi-static and dynamic poroelasticity for fully saturated media were presented by Biot $(1956,1962)$ and are the classical papers on the subject. More recent descriptions of Biot theory in relation to electrokinetic effects are given by Neev and Yeatts (1989), Pride (1994) and Pride and Haartsen (1996). From Biot's theory, pore fluid participates in the rock motion (induced by an acoustic wave) due to viscous friction and inertial coupling. In the steady-state (plane-wave) case, Biot's theory suggests two different mechanisms for converting the acoustic wave oscillation into relative fluid-rock motion thereby allowing the generation of an electrokinetic charge separation. In both cases, the fluid motion can be described as a Darcy particle velocity by analogy with Darcy's law for fluid flow in a porous medium.

The first conventional Biot wave (the so-called fast wave) produces rock and fluid motion which are inphase and the Darcy velocity is determined primarily by fluid viscosity and permeability. Biot theory also indicates that an acoustic wave is partially converted into a 'slow' wave at an interface. The slow wave is highly dispersive and attenuates rapidly on a typical scale of much less than $1 \mathrm{~m}$ (Thompson and Gist, 1993). Despite the rapid attenuation, the slow wave is capable of generating a large Darcy velocity typically greater than that generated by the fast wave through a high permeability formation (Thomson and Gist, 1993). In principle, the dispersive nature of the slow wave implies a capability for high vertical resolution. Although both waves may produce relative rock-fluid displacement capable of generating electrokinetic coupling the effective area and volume contributions will differ in the two cases. In the case of a downgoing hemispherical wavefront incident on a tabular horizon the anticipated electromagnetic response due to electro- kinetic coupling is a vertical electric dipole oscillating at the same frequency as the acoustic source. The area across which the acoustic oscillations are coherent is the first Fresnel zone. The magnitude of the dipolar field is usually expressed by its moment, i.e. the product of current and volume. In the case of fast wave coupling, the volume may be expressed as the product of the area of the first Fresnel zone and the formation thickness. In the case of slow wave coupling the volume would be expressed as the product of the area of the first Fresnel zone and the slow wave attenuation length.

\section{The vertical electric dipole}

According to the description given above, the geophysical signature of electrokinetic coupling due to vertical acoustic wave propagation will be that of a vertical electric dipole (VED) centered directly below the shot point. An important feature of the coupling is that the electromagnetic oscillation will 
propagate to the surface at the speed of light. At a given depth, the time instant of coupling will occur at the one-way transit time of the downgoing acoustic wave. The behaviour of the fields associated with a buried VED can be investigated by numerical modelling. To alleviate the problem of prescribing a field situation, the calculations are done using a point source VED with unit dipole moment. A crosssection through the earth showing contours of the horizontal electric field (Ey) due to a VED at a depth of $10 \mathrm{~m}$ is shown in Fig. 3. The relevant parameters of the model are a frequency of $80 \mathrm{~Hz}$, a uniform halfspace of resistivity $100 \Omega$.m with a relative dielectric constant of unity. In Fig. 3 a banded contour scheme is used to emphasize the field gradients.

The Ey field exhibits dipolar field symmetry about the acoustic source location $(y=0)$ which represents a field null point. Maximum at-surface field magnitudes occur, symmetrically, at $y=-5$ and $+5 \mathrm{~m}$, i.e. at half the depth of the VED source. For a subsurface dipolar source, maximum surface amplitudes will always be observed at an offset equal to half the depth of the source. Equally important is the phase reversal (180 degrees) of the field oscillations occurring about the plane of symmetry $(y=0)$. The phase reversal is highly significant in that all other sources of electromagnetic radiation (natural and anthropogenic), which may interfere with the measurement, are 'distant' and would appear as in-phase oscillations across the 'local' scale of the measurement depicted in Fig. 3.

Although equivalent bipolar magnetic field behaviour is generated by the VED source, the magnitude of the at-surface magnetic field $(\mathrm{Hx})$ oscillations is expected to be very small. In the example shown, the maximum $\mathrm{Hx}$ field is 750 picoTesla. Such fields are too small to be routinely measured with current geophysical field magnetometers. It therefore appears that surface measurements of the horizontal electric field (for which geophysical devices with adequate signal/noise exist) are appropriate for investigations of electrokinetic coupling. The horizontal electric field cannot be measured at a point.
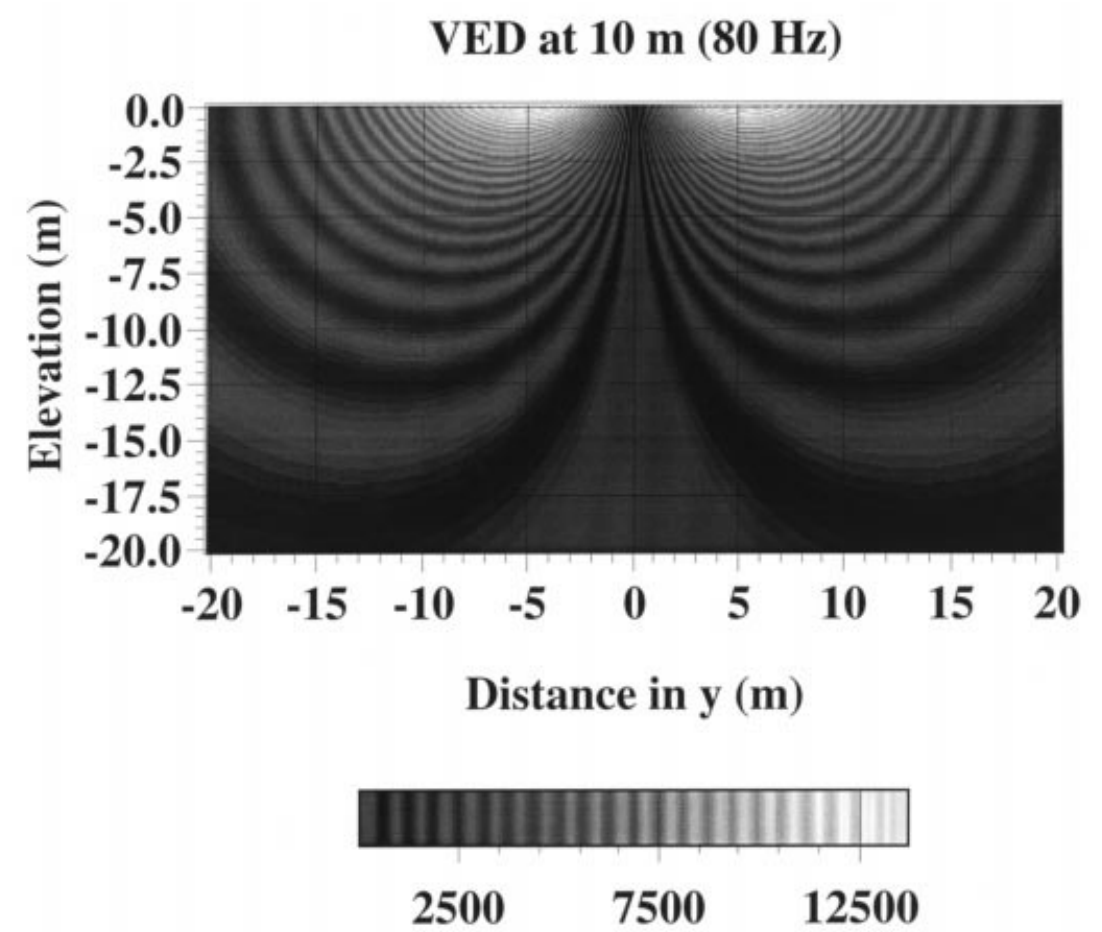

\section{Amplitude in microvolts/m}

Figure 3. A subsurface cross-section showing contours of the amplitude of the horizontal electric field due to a vertical electric field dipole (VED) located at a depth of $10 \mathrm{~m}$. 
The gradient of the measurements might be required to locate a position of maximum field strength and thereby a depth to source, this is not strictly required if an alternative characteristic of electrokinetic coupling is exploited. As described previously, the time-instant of coupling will occur at the one-way transit-time of the down-going acoustic wave. Thus if the acoustic velocity structure is known, or can be estimated, then the time-instant of coupling can be converted to an equivalent depth.

The potential $(\phi)$ across two grounded electrodes is the routine method of measurement since $E=-$ $\Delta \phi$. In practice a very small electrode separation (e.g. $<1 \mathrm{~m}$ ) may result in low signal/noise while a large separation may average the field gradients defining the spatial form of the field. Some form of compromise is inevitable. The problem is studied by extending the analysis of the VED to various depths and examining the signals obtained at various positions along the surface. The locations are best described as a horizontal offset from the source (acoustic shot) position. A common feature of the VED analysis is that the maximum field strength is observed at an offset equal to one-half the depth of the VED source. For the analysis 'virtual electrode separations' of vanishingly small length are assumed.

Figure 4 shows the variation of the horizontal electric field at 4 offset positions $(2,4,10$ and $20 \mathrm{~m})$ for VED depths of between 1 and $50 \mathrm{~m}$. The moment of the VED remains constant ( 1 A.m) at all depths. At first sight the response behaviour of individual offset locations shown in Fig. 4 may appear counterintuitive. Although, as discussed previously, the maximum surface field amplitude is generated at an offset equal to one-half the source depth, for each specific location a maximum response is observed when the source depth is one-half the offset distance.

From Fig. 4 it is evident that small offset locations provide (i) the largest field amplitudes, particularly for near-surface VED sources and (ii) the highest degree of depth sensitivity. For the example used (constant dipole moment with depth), field amplitudes from deeper sources of coupling (e.g. $>20 \mathrm{~m}$ ) will provide similar amplitudes at both near $(2 \mathrm{~m})$ and far $(20 \mathrm{~m})$ offsets. For a VED source at $50 \mathrm{~m}$ depth, the field amplitude at $2 \mathrm{~m}$ offset is reduced by less than one order of magnitude from that at an offset of $20 \mathrm{~m}$. Thus while it is possible to envisage multichannel systems capable of measuring Ey at sequential offsets, the most effective and sensitive measurement location occurs in the immediate vicinity of the shot point. In order to accurately measure the field gradients from possible shallow sources it is clear that very small electrode separations (e.g. 1 or $2 \mathrm{~m}$ ) are required.

Although it might be construed that an extensive array of offset measurements might be required to locate a position of maximum field strength and thereby a depth to source, this is not strictly required if an alternative characteristic of electrokinetic coupling is exploited. As described previously, the timeinstant of coupling will occur at the one-way transit time of the downgoing acoustic wave. Thus if the acoustic velocity structure is known, or can be estimated, then the time-instant of coupling can be converted to an equivalent depth.

\section{Two-channel EK sounding}

The 'two channel' approach to EK sounding forms the basis of a recent commercial geophysical instrument intended for hydrogeological investigations. The instrument and associated interpretation procedures have been widely used overseas, especially in Africa (Millar, 1995). The basic investigation of the EK effect concerns the time- dependent behaviour of the voltages that are recorded by symmetrical surface dipoles. A conceptual model for EK behaviour consists of a 3-layer sub-surface. Layer 1 is an at-surface layer which defines the depth to a fully saturated formation (e.g. an aquifer). If this first layer is impermeable or contains no pore fluids, no EK signal can be generated. Layer 2 comprises a zone from which an EK response may be observed given one or more fluid-filled 
permeable horizons. Layer 3 underlies layer 2 and allows a bedrock/basement to be defined. The layers must be assigned known or estimated seismic velocities and thicknesses in order to convert the recording time of an EK measurement into depth.

Clearly the above conceptual model is simplistic. It refers to a simple two-phase (solid-liquid) medium which is either totally unsaturated (the 'cover' defined by Layer 1) or fully saturated (the 'aquifer' defined by Layer 2). In reality the effects of zones of partial saturation, particularly with regard to conceptual Layer 1, must be taken into account in the interpretation of EK measurements. The seismic pulse passing through a partially saturated material can also generate voltages which contribute to the measurements.

An EK 'signal' may be identified as time-dependent behaviour which is 'in-phase' on the two electric dipoles placed symmetrically about the shot point (the actual polarity of one of the dipoles having been reversed). An EK signal must also be repeatable over successive recordings (hammer shots).

According to the conceptual model, noise (non-EK behaviour) will be observed above (or in the absence of) a subsurface, permeable and saturated horizon. Noise, or non-EK behaviour in the recording, is identified as time-dependent behaviour which is 'independent' across the two channels. The degree to which the time behaviour of the two channels can be identified as 'in- phase' is part of an EK interpretation. In practice an EK sounding (time/voltage measurement) from a single hammer blow forms a shot record. The shot is repeated a sufficient number of times to investigate the repeatability of the sounding and to allow noise and any electromagnetic interference (particularly electrical mains) to be sup-pressed. Individual shot records may be stacked and averaged to allow a final stacked sounding to be obtained.

Our own experiences with two channel EK soundings using a hammer impact source in a variety of hydrogeological settings confirmed a number of facts:

- shot-symmetric, in-phase voltages can be observed in an extremely wide range of soil and sedimentary environments. Peak-to-peak amplitudes, over the first $50 \mathrm{~ms}$ of a recording, typically range from a maximum of $10 \mathrm{mV} / \mathrm{m}$ to less than $0.5 \mathrm{mV} / \mathrm{m}$.

- when shot-symmetric behaviour is observed, the time/voltage behaviour is often highly repeatable both from shot-to-shot (e.g. Figure 5) and from repeated soundings on different days.

- essentially the same behaviour is observed independent of electrode-type (e.g. stainless steel, various non-polarising types $\left(\mathrm{Cu} / \mathrm{CuSO}_{4}, \mathrm{~Pb} / \mathrm{PICl}_{2}\right)$ and lead rods). The voltage returns are not generated by 'electrode shake', i.e. simple mechanical vibration of one or more electrodes.

- the time-signature of the largest voltage returns are consistent with the form of the theoretical seismic wavelet (i.e. a modified sinusoid or 'Ricker' wavelet).

Figure 5 shows a typical two-channel EK recording above a sandstone aquifer capped by $0.5 \mathrm{~m}$ of boulder clay. The water table is at a depth of about $25 \mathrm{~m}$. The sounding was repeated on two different dates. The only significant processing applied to these data is the removal of $50 \mathrm{~Hz}$ mains and its harmonic components. The display uses a polarity convention such that the 180 phase-reversal due to a centered VED is displayed as in-phase behaviour on the two channels. In each case, three individual shot records are shown from two shot-symmetric $2 \mathrm{~m}$ dipoles. The broad form of the soundings is similar on both dates and on each date the individual shot data are highly repeatable. The data on the 
earlier recordings contain some higher frequency components that are not immediately apparent on the second date. In both cases the data across the initial 2-3 ms of the recording display nonelectrokinetic (out-of-phase) behaviour.

The pervasiveness of effects such as those shown in Fig. 5, from a wide variety of hydrogeological environments, together with the lack of a simple signature from the water table, has led to far more extensive field experiments to spatially map the behaviour of both the acoustic and electromagnetic fields in the vicinity of the shot point. At present such experiments are largely confined to at-surface measurements but downhole measurements of the wave effects are anticipated.

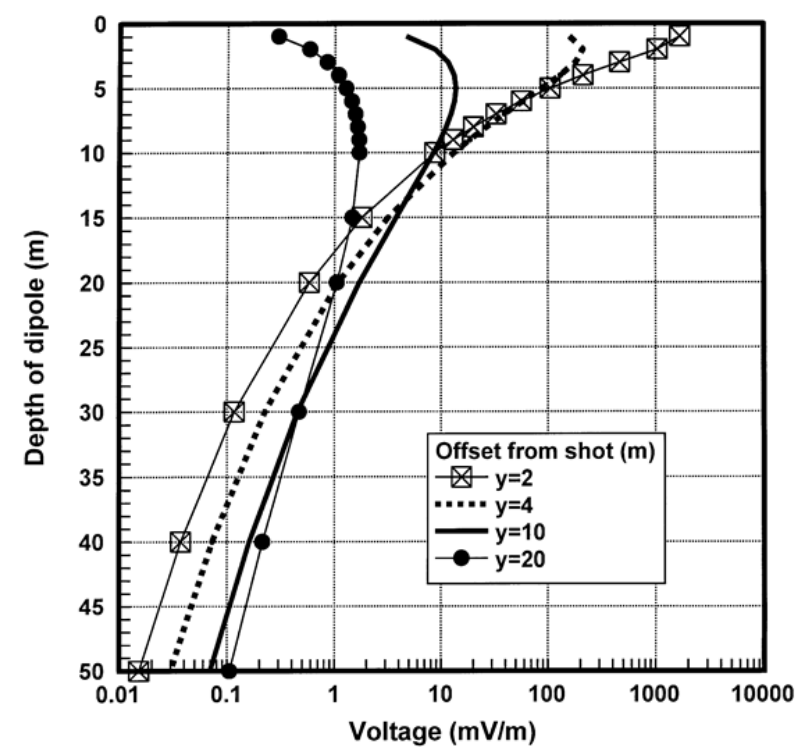

Figure 4. Variation of amplitude of horizontal electric field generated by a buried vertical electric dipole (VED) at depths from 1 to $50 \mathrm{~m}$. 

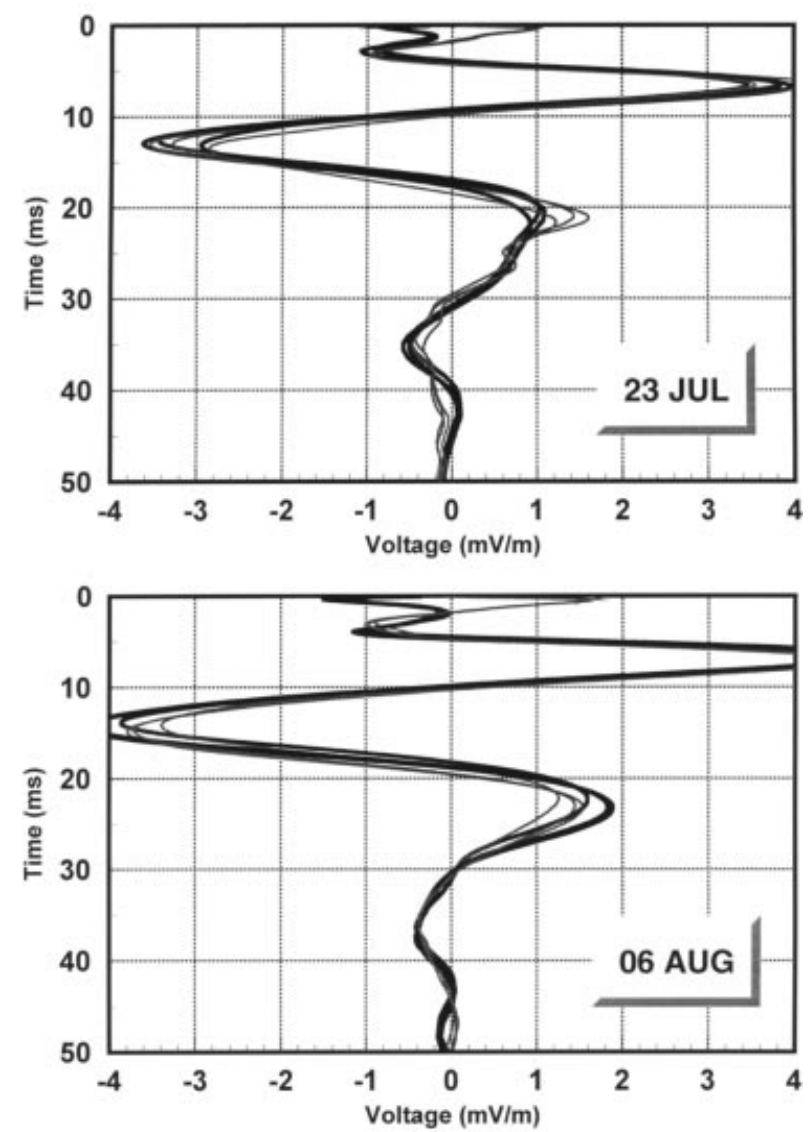

Figure 5. Typical shot-symmetric, two-channel sounding curves above a sandstone aquifer.

\section{Multi-channel EK sounding}

The voltage returns that are observed are clearly associated, in some manner, with the passage of the seismic wave out from the shot point. Clearly the use of only two channels is not discriminatory in terms of describing the complete spatial/temporal nature of the voltages generated. It has been necessary to extend the observations to multi-channel measurements at various offsets from the shot point. This type of 'move out' experiment is com- mon in seismic refraction studies which use an offset spread of geophone receivers to investigate the subsurface acoustic velocity structure. In the case of EK experiments, the seismic geophones are replaced by pairs of E-field dipoles. In practice such move out experiments are duplicated using both geophones and dipoles to allow examination of both acoustic and voltage behaviour. Recent shallow experiments along these lines are reported by Dietrich et al. (1996) and by Butler et al. (1996).

In practice any conventional seismic source when interacting with the sub- surface generates both body and surface waves. The body waves may include compressional and shear components and both refracted and reflected waves can be generated by interfaces and observed at the surface. Surface or Rayleigh waves (e.g. ground roll) are generated along the free surface and are typically low frequency, low velocity waves. This site-dependent complexity of acoustic behaviour has two main geometrical implications for EK experiments. The main implications concern EK coupling due to vertically propagating waves (as discussed previously) and those due to horizontally propagating waves. Two of the main horizontally propagating waves are the surface wave and the critically 
refracted head wave which moves along a subsurface inter- face and generates a charge separation across the boundary (Mikhailov et al., 1997). The EK oscillations associated with both surface and head waves would be detected at increasing times as the waves moved out from the source beneath an array of surface dipoles. This contrasts with instantaneous arrivals to be expected from coupling due to vertical propagation.

The basic question to be addressed is to what extent the shot-symmetric voltages are caused by (i) lateral (horizontal) move out of the acoustic wave and/ or (ii) vertical propagation. Both cases are capable of generating shot-symmetric behaviour (assuming tabular structure). In the first (horizontal propagation) case of surface wave effects, EK coupling would typically generate low frequency voltage oscillations and would be associated with the very low seismic velocities in the uppermost material (typically unconsolidated). Again in the first (horizontal propagation) case of head wave effects, EK coupling would generate voltage oscillations at the compressional wave frequency and be associated with the higher velocity of the material below the head wave interface. In the second (vertical propagation) case, the voltage returns would be associated with the vertical distribution of seismic velocities and would enable a 'vertical sounding' capability. Only in this second case would EK signals (if generated) propagate back from a subsurface horizon and appear as instantaneous events across an array of surface dipoles.

Figure 6 is a summary of 'typical' dual field moveout behaviour observed in the immediate vicinity of a shot point. The experiment is highly detailed (E-field dipole lengths of $1 \mathrm{~m}$ ) with both sets of measurements obtained at intervals of $50 \mathrm{~cm} .10 \mathrm{~Hz}$ geophones and a data acquisition rate of $20 \mathrm{kHz}$ were used. The two sets of results have been treated identically using individual trace normalization (to +1 and -1 ) across the 100 ms time window shown. The geological environment is the Gore sandriver in the Bikita district of southern Zimbabwe.

The seismic data, contoured in the left frame, display time-offset gradients out from the shot point and these gradients define apparent velocities of the propagating waves. There is a high degree of symmetry in the behaviour about the shot point. Standard seismic refraction analysis of the first arrivals indicates a three layer velocity sequence of 180,380 and $1000 \mathrm{~m} \mathrm{~s}^{-1}$ with inter- face depths of 0.6 and $1.3 \mathrm{~m}$. Although there are indications of superimposed effects following the first arrival (first negative/positive going pulse), two further arrivals ( $>40 \mathrm{~ms}$ ) arrivals can be identified having estimated moveout velocities of 400 and $1300 \mathrm{~m} \mathrm{~s}^{-1}$. These velocities are consistent with head waves propagating along the two refractor interfaces.

The voltage data, contoured in the right frame of Fig. 6, appear to reflect the behaviour of the seismic data to some degree. The apparent move out velocity of the first three coherent oscillations (at times $>20 \mathrm{~ms}$ ) is about $325 \mathrm{~m} \mathrm{~s}^{-1}$ and they possess the same dominant frequency (90 $\mathrm{Hz}$ ) as the seismic source. At times in excess of $5 \mathrm{~ms}$, all the voltage oscillations appear to be associated with EK coupling due to the passage of horizontally propagating acoustic waves. At times $<5 \mathrm{~ms}$, however, a high-frequency oscillation (positive/negative/positive) is observed simultaneously across the dipole spread. Converting the onset time of the signal $(2.5 \mathrm{~ms})$ to depth, assuming an upper layer velocity of $180 \mathrm{~m} \mathrm{~s}^{-1}$, yields a depth of $45 \mathrm{~cm}$. This depth is in close agreement with the observed water level in pits dug in the river bed and may therefore be interpreted as electrokinetic coupling at the water table.

The above example is the only occasion where EK voltage returns appear simultaneously across arrays of surface dipoles. The shot-symmetric voltage returns that are typically observed in the vicinity of the shot point (having amplitudes in the millivolt range) appear to be commonly associated with the at- and near-surface horizontal propagation of a variety of seismic waves. Our studies and other recent presentations of joint seismic and voltage data sets (Dietrich et al., 1996; Mikhailov et al., 1997) have 
indicated that, certainly at shallow depths $(<10 \mathrm{~m})$, the two basic types of electrokinetic conversion may occur and be observed. The first type, involving vertical acoustic wave propagation, results in simultaneous voltage returns across arrays of surface dipoles. This type of conversion, where it occurs, would enable vertical sounding of subsurface properties. In our experience, simple evidence for this type of conversion has been difficult to establish due to the dominance of the second type of conversion. As discussed above, in many instances the voltages that appear in such experiments have arrival times that increase with dipole offset and appear to be associated with a variety of acoustic waves propagating horizontally in the near-surface environment.
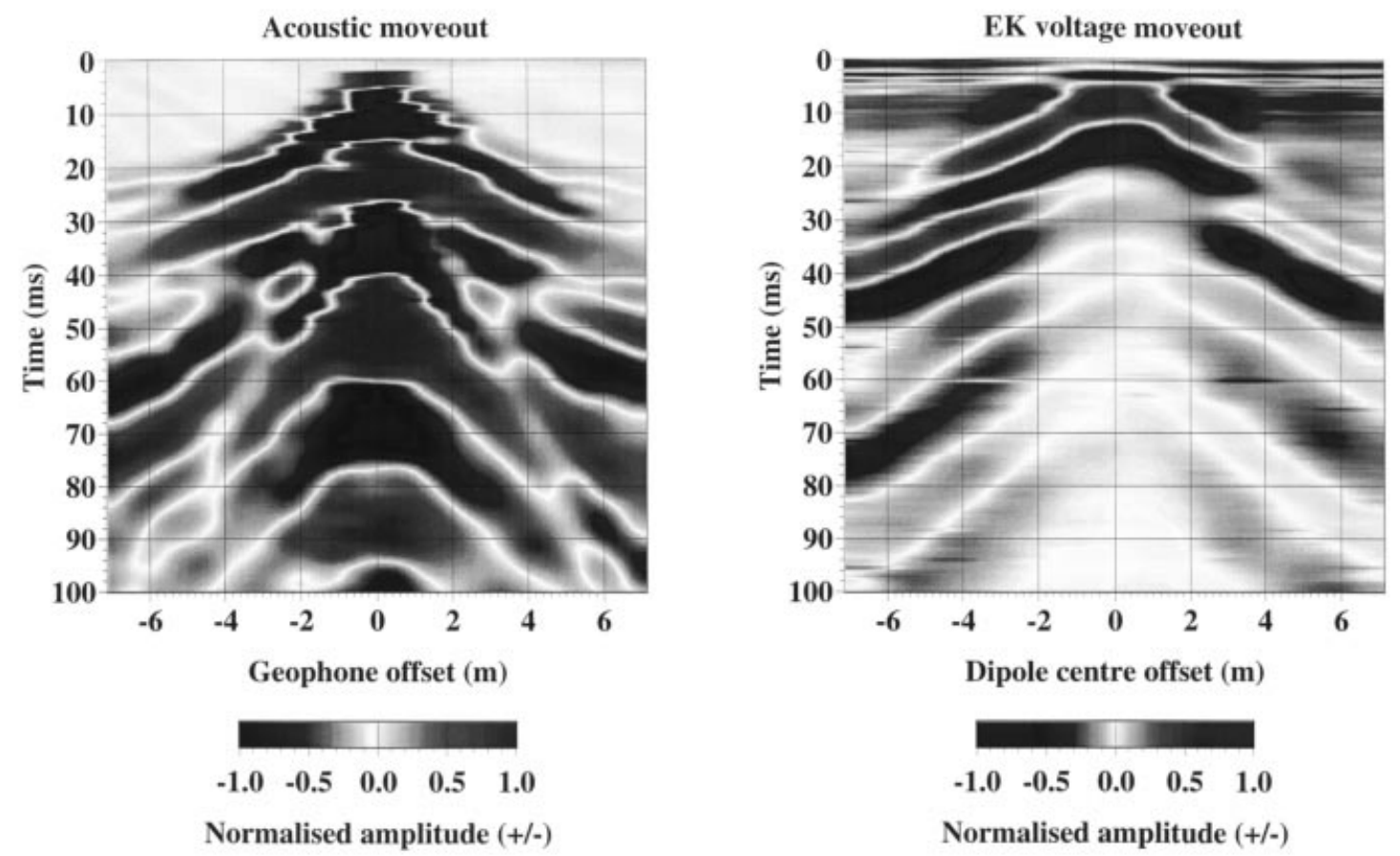

Figure 6. Detailed joint (acoustic and voltage) moveout experiment from the Gore sand river in southern Zimbabwe.

Observational joint data sets that address the basic questions of electrokinetic effects are renewing interest in the subject since, from recent theoretical predictions (Neev and Yeatts, 1989; Pride and Haartsen, 1996), it offers direct insight into subsurface fluids and the porosity and permeability of rocks. Combining, as it does, the macroscopic behaviour of both acoustic and electromagnetic wavefields, together with the geochemistry and electrical rock properties at the pore scale, the subject offers rich scope for further investigation.

\section{Acknowledgements}

Some of the work described here was supported by the Department for International Development (TDR Project R6232). The views expressed are not necessarily those of DFID. This paper is published with the approval of the Director, British Geological Survey (NERC).

\section{References}

Ahmed, M.U., 1964. A laboratory study of streaming potentials. Geophys. Prosp., 12, 49-64.

Biot, M.A., 1956. Theory of propagation of elastic waves in a fluid saturated porous solid, I. Low-frequency range. J. Acoust. Soc. Am., 28, 168-178. 
Biot, M.A., 1962. Mechanics of deformation and acoustic propagation in porous media. J. Appl. Phys., 33, 1482-1498.

Bockris, J. and Ready, A.K.N., 1970.

Modern Electrochemistry. Plenum Press.

Broding, R.A., Buchanan, S.D. and Hearn, D.P., 1963. Field experiments on the electroseismic effect. IEEE Trans. Geosci. Electronics, GE-1, 23-31.

Butler, K.A., Russell, R.D., Kepic, A.W. and Maxwell, M., 1996. Measurement of the seismoelectric response from a shallow boundary. Geophysics, 61, 1769-1778.

Corwin, R.F., 1990. The self-potential method for environmental and engineering applications. In: Geotechnical and Environmental Geophysics (S.H. Ward, ed.), Vol. 1, 127-145. Society of Exploration Geophysics, Tulsa.

Corwin, R.F. and Hoover, D.B., 1979. The self-potential method in geothermal exploration. Geophysics, 44, 226-245.

Debye, P., 1933. A method for the determination of the mass of electrolyte ions. J. Chem. Phys., 1, 13.

Dietrich, M., Garambois, S. and Glangeaud, F., 1996. Seismo-electric effects: a field example over a shallow aquifer. Proc. EEGS 2nd Ann. Mtng, Nantes, France, 1996, 82-84.

Fitterman, D.V., 1978. Electrokinetic and magnetic anomalies associated with dilatant regions in a layered earth. J. Geophys. Res., 83, 5923-5928.

Ishido, T. and Mizutani, H., 1981. Experimental and theoretical basis of electrokinetic phenomena in rock-water systems and its applications to geophysics. J. Geophys. Res., $86,1763-1775$.

Ivanov, A.G., 1939. Effect of electrization of earth layers by elastic waves passing through them (in Russian). Dokl. Akad. Nauk SSSR, 24 (1), 41-45.

Jouniaux, L. and Pozzi, J.P., 1995. Permeability dependence of streaming potential in rocks for various fluid conductivities. Geophys. Res. Lett., 22, 485-488.

Lageman, R., Pool, W. and Seffinga, G., 1989. Electro-reclamation theory and practice. Chem. Ind. London, 18, 575-579.

Long, L.T. and Rivers, W.K., 1975. Field measurement of the electroseismic response. Geophysics, 40, 233-245.

Martner, S.T. and Sparks, N.R., 1959. The electroseismic effect. Geophysics, 24, 297-308.

Mikhailov, O.V., Haartsen, M.W. and Toksoz, M.N., 1997. Electroseismic investigation of the shallow subsurface: Field measurements and numerical modeling. Geophysics, 62, 97-105. 
Millar, J., 1995. A source of life made commercial. Physics World, October 1995, 22-23.

Mizutani, H.T., Ishido, T., Yokokura, T. and Ohnishi, S., 1976. Electrokinetic phenomena associated with earthquakes. Geophys. Res. Lett., 3, 365-368.

Murty, Y.S., 1985. First results on the direct detection of groundwater by seismoelectric effect - a field experiment. Bull. Austr. Soc. Expl. Geophys., 16, 254-255.

Neev, J. and Yeatts, F.R., 1989. Electrokinetic effects in fluid-saturated poroelastic media. Phys. Rev. B., 40, 9135-9141.

O'Brien, R.W., 1988. Electro-acoustic effects in a dilute suspension of spherical particles. J. Fluid Mech., 190, 71-86.

Overbeek, J.Th.G., 1952. Electrochemistry of the double layer. In: Colloid Science, Vol. 1, Irreversible Systems (H.R. Kruyt, ed.), pp. 115-193. Elsevier, New York.

Parkhomenko, E.I., 1971. Electrification Phenomena in Rocks. Plenum Press, New York.

Parkhomenko, E.I. and Gaskarov, I.V., 1971. Borehole and laboratory studies of the seismoelectric effect of the second kind in rocks. Izv. Akad. Sci. USSR, Physics Solid Earth, 9, 663-666.

Pride, S.R., 1994. Governing equations for the coupled electromagnetic and acoustics of porous media. Phys. Rev. B., 50, 15, 678-15,696.

Pride, S.R. and Haartsen, M.W., 1996. Electroseismic wave properties. J. Acoust. Soc. Am., 100, 13011315.

Pride, S.R. and Morgan, F.D., 1991. Electrokinetic dissipation induced by seismic waves. Geophysics, 56, 914-925.

Probstein, R.F. and Hicks, R.E., 1993. Removal of contaminants from soils by electric fields. Science, 260, 498-503.

Revil, A., Darot, M. and Pezard, P.A., 1996. From surface electrical properties to spontaneous potentials in porous media. Surv. Geophys., 17, 331-346.

Shapiro, A.P. and Probstein, R.F., 1993. Removal of contaminants from saturated clay by electroosmosis. Environ. Sci. Tech., 27, 283-287.

Thompson, R.R., 1936. The seismic-electric effect. Geophysics, 1, 327-335.

Thompson, A.H. and Gist, G.A., 1993. Geophysical applications of electrokinetic conversion. The Leading Edge, 12, 1169-1173. 